\title{
Temperature profiles with bi-static Doppler-RASS and their correction
}

\author{
B. Hennemuth ${ }^{1}$, G. Peters ${ }^{2}$, and H.-J. Kirtzel ${ }^{2}$ \\ ${ }^{1}$ Consulting Meteorologist, Hamburg, Germany \\ ${ }^{2}$ METEK Meteorologische Messtechnik GmbH, Elmshorn, Germany \\ Correspondence to: B. Hennemuth (barbara.hennemuth@zmaw.de)
}

Received: 2 December 2011 - Published in Atmos. Meas. Tech. Discuss.: 3 February 2012

Revised: 24 May 2012 - Accepted: 25 May 2012 - Published: 22 June 2012

\begin{abstract}
The technique of atmospheric temperature profiling by Doppler-RASS is discussed. The set up with bi-static (separated transmit and receiving) antennas implies a range dependent scattering angle. The retrieval scheme developed by Kon for such antenna geometry is reviewed and its limits of validity are discussed. Empirical tuning of the effective antenna aperture is proposed to fit the retrieved temperature profiles to reality. The method is based on the assumption that potential temperature profiles under presumedly neutral conditions are constant with height. Examples of application of the measuring technique for atmospheric boundary layer characterization are presented.
\end{abstract}

\section{Introduction}

Temperature profiling in the lower troposphere is of great importance for several applications, e.g. for air pollution dispersion issues, determination of boundary layer height and of stratification in the mixing layer, or climatology of temperature profiles beyond $2 \mathrm{~m}$, which is needed for validation of atmospheric models. For such applications continuous measurements of temperature gradients are needed. While atmospheric stability is treated as a global variable in traditional routine air quality monitoring concepts, modern numerical models are able to account for the real local nature of stability and can accommodate even complex temperature profiles. RASS (Radio Acoustic Sounding System) has proven to be an adequate measuring system for high resolution temperature profiles in various atmospheric height ranges depending on the design characteristics.
It uses the scattering of radio waves from acoustic wave fronts to measure the speed of sound. This sound speed is translated into the so called sonic temperature, which is very near to the virtual temperature (Kaimal and Gaynor, 1991) and therefore controlling the hydrostatic stability.

The principles of RASS technology were developed and demonstrated already four decades ago (e.g. Marshall et al., 1972; North et al., 1973; Nalbandian, 1977; Makarova, 1980), and systematic comparisons of RASS temperature profiles with in-situ soundings, radiometric soundings or mast measurements were accomplished (e.g. Bonino et al., 1985; Angevine et al., 1998; Görsdorf, 1998; Argentini et al., 2008; Pérez et al., 2008). In early experiments pulses of sound waves were used with fixed frequency that was tuned for maximum amplitude of the scattered radio waves. This frequency $f_{\mathrm{B}}$ is said to satisfy the "Bragg-condition" according to Eq. (1)

$f_{\mathrm{B}}=c_{a} \frac{2 \sin \frac{\alpha}{2}}{\lambda_{e}}$

where $c_{a}, \alpha$ and $\lambda_{e}$ are the sound velocity, scattering angle and length of the radio waves respectively. Due to the atmospheric temperature profile the sound velocity is generally height dependent. Therefore, the Bragg condition can be satisfied only in a particular height. Although the scattered signal may be still above the receiver detection threshold despite some deviation of the sound frequency from $f_{\mathrm{B}}$, the relation between the observed frequency shift and the sound velocity becomes ambiguous, and the interpretation in terms of Doppler shift would lead to false temperature gradients (Kon and Tatarskii, 1980). A comprehensive review on this issue can be found in Kallistratova and Kon (1985) (in Russian language). In the further course of exploring the method, 
two classes of RASS evolved, which use either the propagation time of the electromagnetic waves (Bragg-RASS) or of the acoustic waves (Doppler-RASS) for range discrimination (Peters et al., 1983). In both cases the above mentioned problem is eliminated by transmitting frequency modulated acoustic signals with a bandwidth embracing the Bragg condition within the total sounding height range.

Later, various corrections have been developed which account for cross sensitivities to atmospheric parameters like horizontal and vertical wind, turbulence, stratification, and humidity as well as to geometric features of the radio and the acoustic antennas (Lataitis et al., 1993; Kon, 1985; Peters and Angevine, 1996; Petenko, 1999). While these corrections are common for both classes of RASS, a systematic distortion of low level temperature gradients, especially observed with Bragg-RASS, was successfully analyzed by Görsdorf and Lehmann (2000) and an efficient correction scheme was proposed.

Here we consider the Doppler-RASS, which is implemented as a supplement of Doppler-SODAR, and which is primarily used for sampling the lower few hundred meters of the atmosphere. Typically these systems use separated antennas for the continuous transmission and the reception of radio waves (bi-static antenna configuration). This implies that the scattering angle becomes height dependent. Since the effect is most pronounced at short ranges, the usual far field approximation is not applicable for calculating the Doppler shift. The bi-static effect on the relation between Doppler shift and phase velocity of the acoustic waves has been analyzed theoretically by Kon (1981) and a first order near field correction has been derived. Among other approximations a Gaussian illumination distribution of the antenna apertures and isotropic acoustic transmission is assumed for the sake of mathematical convenience. Therefore, a kind of empirical adjustment of algorithm parameters needs to be applied in order to minimize the bias of RASS-derived temperatures at low altitudes.

This paper is organized as follows. The RASS and antenna configurations that were used in this study are described in the Sect. 2, the relation between Doppler shift and phase velocity according to Kon's approximation is introduced in Sect. 3, and a possible bi-static correction of this approximation is proposed in Sect. 4. Finally, results from various measurement campaigns are presented and discussed in Sect. 5 .

\section{The RASS system}

\subsection{Basic principle}

The sound velocity $c_{a}$ is derived from the measured Doppler frequency shift $\delta f$ of the backscattered electromagnetic signal. From the sound velocity, the local air temperature can by inferred. Within ideal gas approximation, the relation between the so called sonic temperature $T_{\mathrm{S}}$ and sound velocity is given by the numerical-value equation
$\frac{T_{\mathrm{s}}}{\mathrm{K}}=\left(\frac{c_{a}}{\mathrm{~m} \mathrm{~s}^{-1}} \frac{1}{20.047}\right)^{2}$.

$T_{\mathrm{S}}$ is related to the temperature by

$T_{\mathrm{S}}=T\left(1+0.32 \frac{e}{p}\right)$

with $e$ water vapor partial pressure and $p$ atmosphere pressure (Kaimal and Gaynor, 1991). $T_{\mathrm{S}}$ is very close to the virtual temperature $T_{\mathrm{v}}$, namely $T_{\mathrm{S}}=T_{\mathrm{v}}(1-0.06 e / p)$. Therefore, the gradient of $T_{\mathrm{S}}$ is a good proxy variable for the static stability of the atmosphere. A comprehensive introduction into the theory of RASS including various cross sensitivities and second order effects can be found in Lataitis (1992).

\subsection{Integrated SODAR/RASS}

For the measurements reported below, a SODAR/RASS manufactured by METEK Ltd. (MERASS) was used. An electro-magnetic continuous-wave transmitter at $1290 \mathrm{MHz}$ and a corresponding receiver is installed adjacent to the SODAR-antenna. METEK-SODARs transmit sound pulses in cycles of up to 5 beam directions. This transmit cycle is extended with an additional RASS-sound-pulse. The SODAR signal processing hardware handles the corresponding RASS receiving signal like an additional beam direction. Thus, RASS- and SODAR-profiles can be measured in nearly any staggered order, such that after averaging of a larger number of cycles the mean RASS- and SODARprofiles can be considered to be quasi-simultaneous. After mixing of the RASS receiving signal into the base band its properties are very similar to SODAR-echoes, such that the same hardware can be used for processing the SODAR- as well as the RASS-echoes. Thus, MERASS is a virtually integrated system for the simultaneous measurement of wind and temperature profiles. With MERASS the temperature profile is measured with a height resolution of down to $10 \mathrm{~m}$ starting at $35 \mathrm{~m}$ above the ground.

\section{Bi-static correction}

Due to the continuous operation of the electromagnetic transmitter, the transmitting and receiving antennas need to be separated as illustrated in Fig. 1. The distance between the antennas of MERASS is typically $4-6 \mathrm{~m}$. Therefore, the scattering angle is not exactly $180^{\circ}$, and it depends on height. Disregarding the bi-static deviation from backscattering would lead to a temperature bias in the order of $1 \mathrm{~K}$ at the lowest altitude, and it would vanish rapidly with increasing height. For some applications, as for example estimating the static stability, the temperature gradient is more important than the absolute temperature itself, and a height dependent bias would lead to significant misinterpretations. 


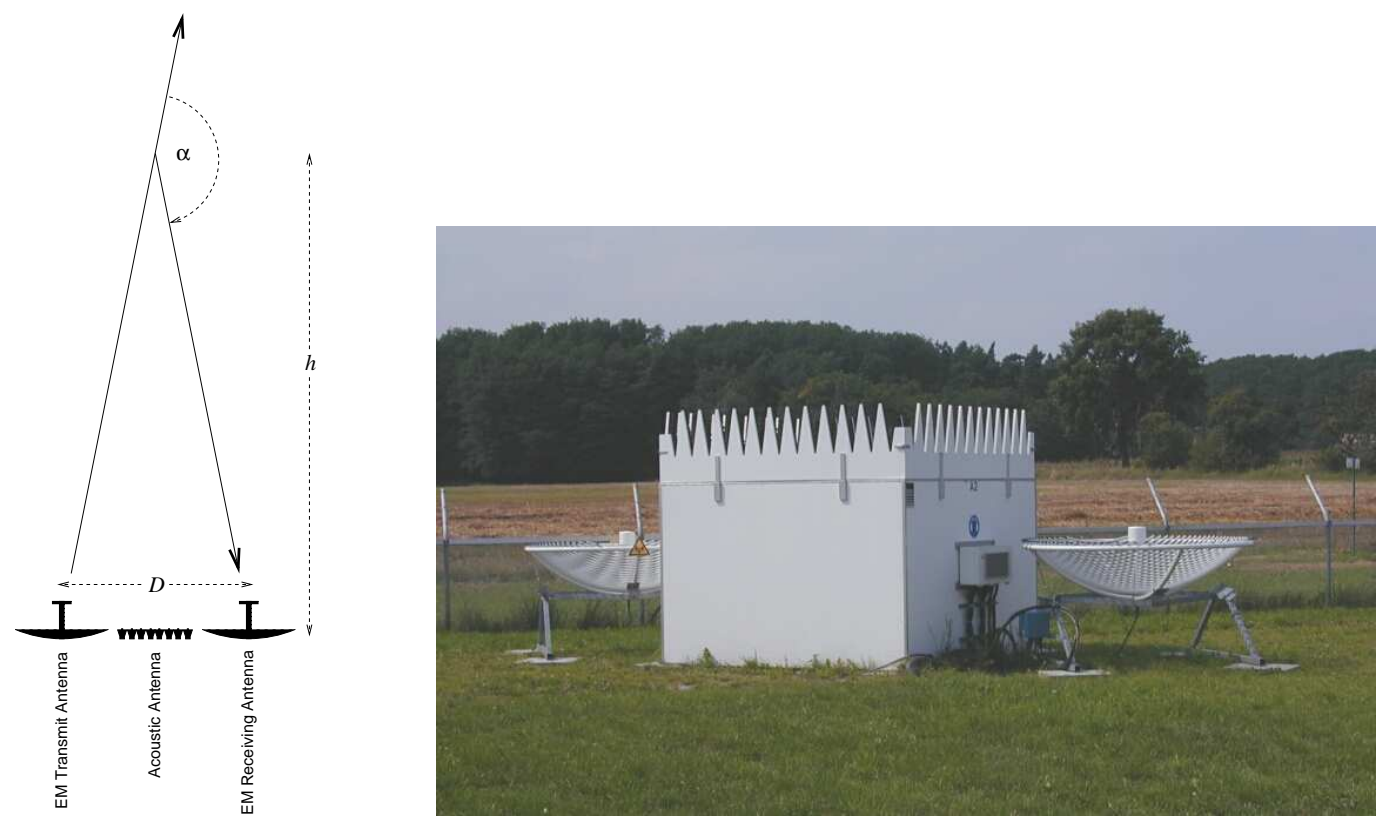

Fig. 1. Bi-static RASS antenna set up. $\alpha$ scattering angle, $h$ measuring height, $D$ distance between electromagnetic transmit and receiving antennas.

For arbitrary scattering angles $\alpha$, the Doppler shift $\delta f_{\text {bi }}$ is given by

$\delta f_{\mathrm{bi}}=2 f_{e} \frac{c_{a}}{c_{e}} \sin \frac{\alpha}{2}$

while for backscattering $\left(\alpha=180^{\circ}\right)$ we have

$\delta f_{\text {back }}=2 f_{e} \frac{c_{a}}{c_{e}}$.

Disregarding the bi-static deviation means to retrieve $c_{a}$ from Eq. (5) but using the Doppler shift $\delta f_{\text {bi }}$, hence

$c_{a}^{*}=c_{a} \sin \frac{\alpha}{2}$

with $c_{a}$ and $c_{a}^{*}$ true and biased sound velocity respectively. With Eq. (2) we obtain for the bias and true temperature

$T=T^{*}\left(\sin \frac{\alpha}{2}\right)^{-2}$.

If the transmitter and receiver were a point-source and -sink respectively, the scattering angle $\alpha$, as indicated in Fig. 1, would be related to the measuring height $h$ and the distance $D$ between transmit and receiving antenna according to

$\tan \alpha=-\frac{D}{2 h}$.

For scattering angles with small deviation from $180^{\circ}$ Eq. (7) would then take the approximate form

$T=T^{*}\left(1+\frac{D^{2}}{4 h^{2}}\right)$.
In reality the extension of the antenna apertures must not be neglected, particularly at low ranges. Here the wavefronts are not perfectly spherical as in the far field. Kon (1981) developed for this range a generalization of Eq. (9) which takes into account the extension of antenna apertures. For mathematical convenience he replaced the real illumination function of the antenna apertures by a rotation-symmetric Gaussian function, which depends only on the distance from the aperture center (see Fig. 2). The standard deviation of the Gaussian function is indicated by $a_{e}$ and a dimensionless farfield parameter $Q_{e}=h /\left(k_{e} a_{e}^{2}\right)$ normalized with the wave number $k_{e}$ is introduced. Kon derived an approximate analytical expression for the bi-static bias analogue to Eq. (7) which accounts for terms up to the order $Q_{e}^{-4}$, and which still assumes only small deviations from backscatter geometry. A further simplification in Kon's model is the assumption of an acoustic point source (isotropic). With these assumptions the correction according to Kon reads

$T=T^{*}\left(1+\frac{1}{4} \frac{D^{2}}{h^{2}} \frac{1-Q_{e}^{-2}}{\left(1+Q_{e}^{-2}\right)^{2}}\right)$.

\section{Proposed semi-empirical correction}

\subsection{Effect of bi-static correction}

Equation (10) does not in all cases give satisfactory results for the lower heights of a RASS temperature profile - even if we restrict the analysis to heights where Eq. (10) should 


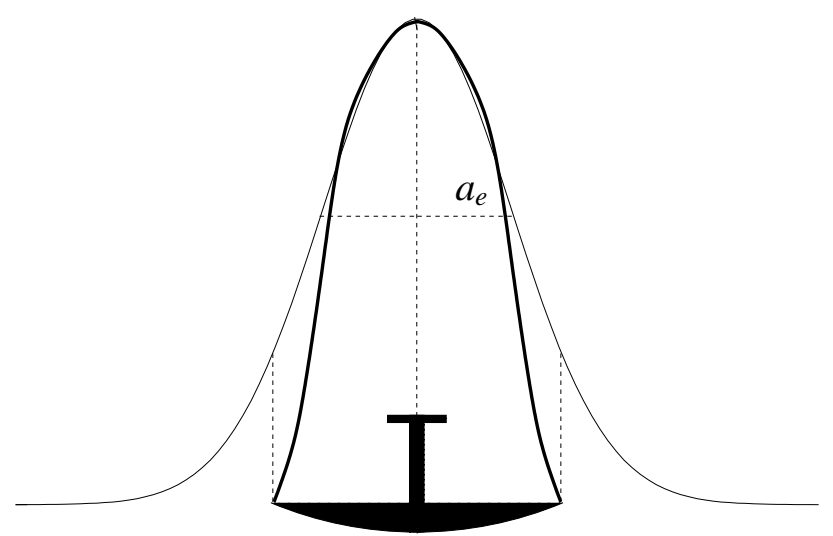

Fig. 2. Real (thick line) and modeled (thin line) Gaussian aperture illumination function with standard deviation $a_{e}$.

be applicable (i.e. $\alpha \approx 180^{\circ}, Q_{e}^{2} \gg 1$ ). This has been found in comparisons with in-situ measurements (Argentini et al., 2008), but also by assessing the coincidence between insitu measured temperature profiles near the surface and the RASS-profiles which start at $30 \mathrm{~m}$ or $40 \mathrm{~m}$ above ground. Equation (10) formula assumes a Gaussian illumination distribution of the antenna apertures, which has by definition an infinite extension, whereas a realistic illumination function is truncated at the physical rim of the aperture. Therefore, there is some arbitrariness in the choice of $a_{e}$, and various approaches are imaginable. Argentini et al. (2009) achieved the best agreements with profiles from tethersondes by choosing an effective radius of $0.6 r$ with $r$ geometric antenna radius. Similarly, the nominal separation of the antennas, defined by the distance of the (Gaussian) beam axes, may not provide the best correction with Kon's model.

We suggest a semi-empirical adaptation of $a_{e}$ and $D$, such that mean RASS temperature profiles under presumedly neutral conditions show constant potential temperature.

The effect of different values of $a_{e}$ and $D$ on the temperature correction $T-T^{*}$ according to Eq. (10) is illustrated in Fig. 3. In this figure, we assume a sound velocity of $340 \mathrm{~m} \mathrm{~s}^{-1}$ (corresponding $14.5^{\circ} \mathrm{C}$ ), a frequency of $1290 \mathrm{Mhz}$, and a physical antenna radius of $1 \mathrm{~m}$. The colors indicate different choices of $a_{e}$ between 0 and $1 \mathrm{~m}$. For each $a_{e}$ three choices of $D$ are shown, as indicated on the corresponding line. A nominal antenna separation of $D=6 \mathrm{~m}$ was assumed, and in addition, the graphs for $\pm 0.5 \mathrm{~m}$ deviation from the nominal separation are plotted. These deviations may be attributed partly to model simplifications and partly to uncertainties of the antenna positioning. The left panel shows the temperature correction, and the right panel shows the corresponding correction of temperature gradient, if it is derived from temperature differences between heights separated by $30 \mathrm{~m}$.

We recognize (except for $a_{e}=0$ ) a height of maximal correction $h_{\max }$ which moves upward with increasing $a_{e}$
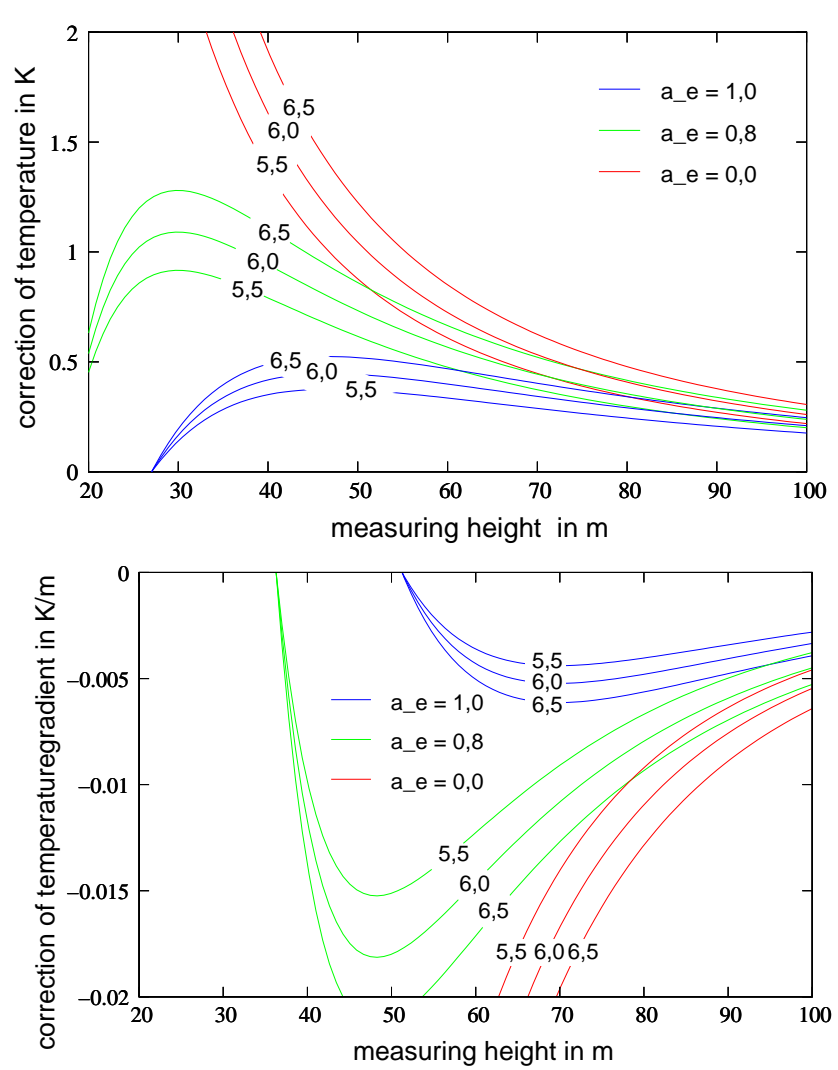

Fig. 3. RASS temperature correction versus height after Kon for different values of $a_{e}$ and $D$. The line-color indicates $a_{e}$. For each color there are three lines, each representing a value of $D$ as indicated in the lines. All lengths in $\mathrm{m}$. The left panel shows the absolute temperature correction. The right panel shows the gradient correction, if it is derived from the temperature difference between two heights with $30 \mathrm{~m}$ separation centered around the indicated height.

$\left(h_{\max } \approx 30 \mathrm{~m}\right.$ for $a_{e}=0.6$ and $h_{\max } \approx 45-50 \mathrm{~m}$ for $\left.a_{e}=1\right)$. Although Kon (1981) did not provide an estimate of the residual correction related to the approximations of his model, we believe that $h_{\max }$ is below the applicable height of Kon's model.

If we set $a_{e}$ equal to the physical radius of the aperture $\left(a_{e}=1 \mathrm{~m}\right)$, the correction proposed by Kon (1981) provides a maximum temperature correction of only $0.52 \mathrm{~K}$. Replacing the physical radius by a reduced effective radius $a_{e}<1 \mathrm{~m}$ increases the temperature correction, and shifts the lower border of the model to lower heights. This offers the possibility to adjust the temperature profile in the near range empirically by the choice of $a_{e}$. The sensitivity of the profile shape to $D$ is comparably small for reasonable variations of $D$. Since the reference measurements available for this study were not sufficiently detailed to provide guidance for the choice of $D$, the nominal value of $D$ is used here, and only $a_{e}$ is varied for adaption to reference measurements. 


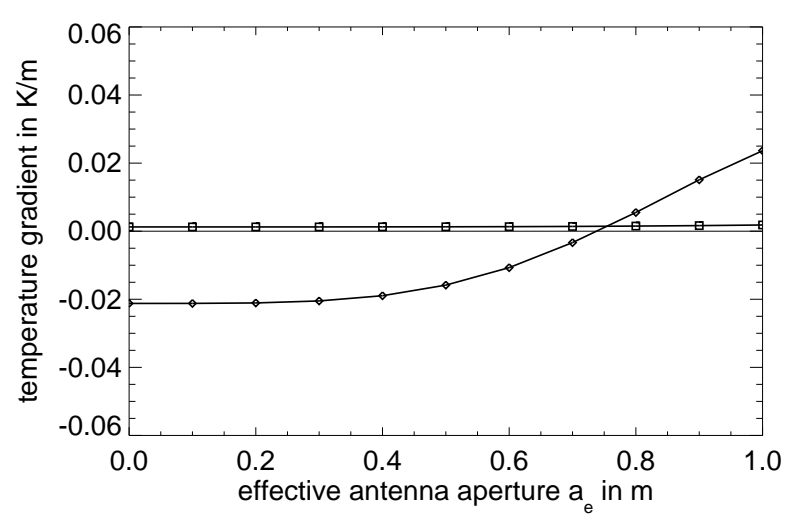

Fig. 4. Median of corrected temperature gradients $\Delta T / \delta h$ as a function of effective antenna aperture $a_{e}$. Gradients are shown at lowest height interval ( $60 \mathrm{~m}-40 \mathrm{~m}$, diamonds) and at elevated height interval (140 m-120 m, squares) during daytime for Munich.

\subsection{Tuning of $a_{e}$ in neutral conditions}

Here we analyse a measuring campaign at Munich Airport north of the city. The campaign took place from 23 June 2010 to 6 October 2010. The site is flat. RASS-temperature data are available every $10 \mathrm{~min}$ and the height levels are $40 \mathrm{~m}$ to $500 \mathrm{~m}$ with $\Delta h=20 \mathrm{~m}$. The distance between the radar antennas is $6 \mathrm{~m}$, and the physical radius of the antennas is $1 \mathrm{~m}$. Near-surface temperature (Pt100-thermometer) and relative humidity were measured at $2 \mathrm{~m}$ and $10 \mathrm{~m}$ height synchronous to the RASS measurements to provide virtual temperature. During the times of flight operations (07:00-23:00 UTC) the corresponding sector $\left(180-360^{\circ}\right)$ is excluded. Nevertheless, data might be influenced by the nearby runway.

The effect of different values of $a_{e}$ on the temperature gradient at the lower heights is investigated by the median of the frequency distribution of the corresponding gradients as a measure for the correction. It is shown in Fig. 4 for values of $a_{e}$ between 0 and 1 for the layers $40-60 \mathrm{~m}$ and $120-140 \mathrm{~m}$. The selected period of the day comprises 09:00-11:00 UTC and 15:00-17:00 UTC, thus excluding possibly stable and unstable conditions. At the elevated height interval the temperature gradient is zero for all $a_{e}$ within an assumed uncertainty range of $\pm 0.005 \mathrm{~K} \mathrm{~m}^{-1}$. This implies that the corrected profiles do not depend on the choice of $a_{e}$. In contrast with this, the median of the gradients at the lowest height interval strongly depends on $a_{e}$. At a value of approximately $0.8 \mathrm{~m}$, a neutral stability gradient is achieved. Therefore, the effective radius $a_{e}$ is set to this value.

\subsection{Validation}

A firm validation by a reference profile is not possible because simultaneous mast measurements are not available. But in most cases the RASS profiles are supplemented by near-surface measurements at $2 \mathrm{~m}$ and $10 \mathrm{~m}$ height which enables a plausibility examination of the constructed complete
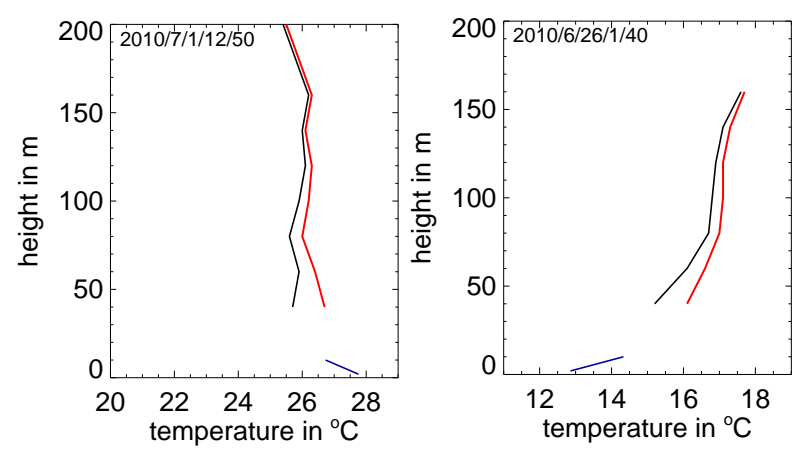

Fig. 5. Temperature profiles at Munich airport without (black) and with corrections (red).

profile. Figure 5 shows temperature profiles around noon and around midnight. The correction is significant in the lower height ranges. The uncorrected stable gradient in the lowest layer at noon is changed to a slightly unstable one, and the weakening of the stable stratification during the night appears to fit the near-surface profiles better. Nevertheless, the large height gap between the the near-surface measurements and the lowest RASS measuring height makes reliable conclusions difficult.

Analysing the whole of temperature gradient by frequency distributions for the lowest two RASS levels and for elevated levels shows (Fig. 6) analogue results. Around noon, there is a clear shift at the lowest levels from mostly positive gradients, i.e. stable stratification (uncorrected) to near-zero and negative gradients, i.e. neutral and unstable stratification (corrected). Around midnight, the gradients are shifted from strongly stable (uncorrected) to moderately stable stratification (corrected). This result seems to be plausible. At larger heights the correction nearly vanishes (Fig. 6, right), which is in agreement with Fig. 4 where a possible correction shows no effect.

Another plausibility test of the bi-static correction according to Kon (1981) is the comparison with a simple empirical correction, which assumes near-neutral conditions - and thus adiabatic temperature profiles. In this approach we assume that this condition is satisfied for profiles measured between 10:00-18:00 UTC with wind speed exceeding $2 \mathrm{~m} \mathrm{~s}^{-1}$. All temperature values $T$ at heights below $150 \mathrm{~m}$ are converted locally to potential temperature $\Theta$ by $\Theta=T+h \cdot \gamma$ with $\gamma=10^{-2} \mathrm{~K} \mathrm{~m}^{-1}$. For all temperature profiles, which match the above conditions, the difference to the $140-\mathrm{m}$ temperature (RASS temperature level below $150 \mathrm{~m}$ ) is determined and averaged. The result is an empirical adiabatic correction for each height lower than $150 \mathrm{~m}$. Assuming that this correction is not restricted to neutral conditions but generally valid, it is applied to all data sets. Figure 7 shows the same two profiles as displayed in Fig. 5. The corrections below $150 \mathrm{~m}$ are nearly identical in both figures, which supports the bi-static correction according to Kon (1981) as being physically reasonable. 

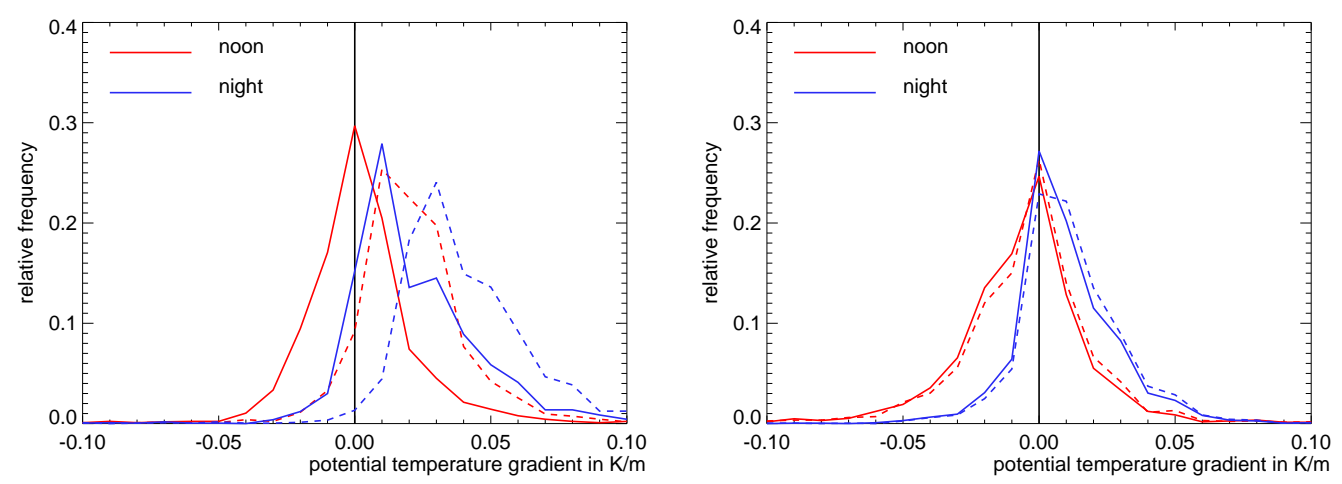

Fig. 6. Frequency distribution of quotient $\Delta T / \delta h$ at lowest height interval $(60 \mathrm{~m}-40 \mathrm{~m}$, left) and at elevated height interval ( $140 \mathrm{~m}-120 \mathrm{~m}$, right) around noon and midnight for Munich. Dashed lines: uncorrected temperature, solid lines: corrected temperature.
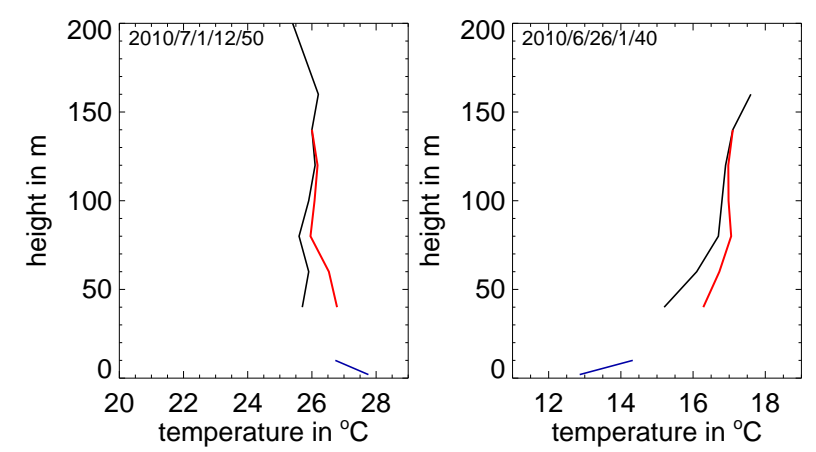

Fig. 7. Temperature profiles at Munich airport without (black) and with (red) empirical adiabatic corrections

\section{Application examples}

We discuss here the physical plausibility of examples of corrected RASS temperature profiles together with supplementary near-surface in-situ measurements.

\subsection{Temperature profile evolution over flat terrain}

The flat environment around Munich Airport was chosen for studying the diurnal boundary layer evolution up to $500 \mathrm{~m}$. Figure 8 shows the evolution within the potential temperature profiles over a $24 \mathrm{~h}$ period in July. During night time the near-surface temperature decreases continuously resulting in a strongly stable stratification, particularly around midnight. In the morning a very rapidly growing neutral boundary layer evolves with top height near 100 m at 07:00 UTC and above the RASS range of $350 \mathrm{~m}$ at 09:00 UTC. The stratification above $50 \mathrm{~m}$ remains neutral until 19:00 UTC, followed by cooling in the lower $50 \mathrm{~m}$.

An explanation of this development can be given by simultaneous measurements of wind speed and sensible heat flux from the sonic anemometer/thermometer at $10 \mathrm{~m}$ height (Fig. 9). The night is rather calm with wind speed mostly lower than $1 \mathrm{~m} \mathrm{~s}^{-1}$, and the weak mixing results in a very

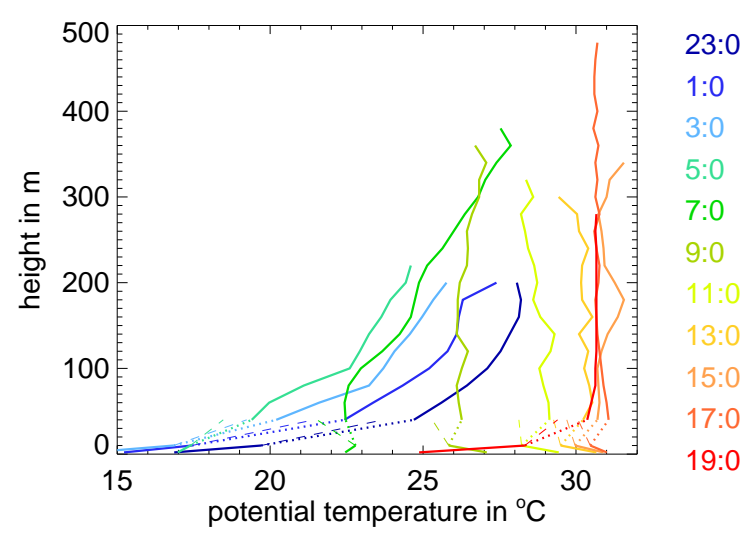

Fig. 8. Potential temperature profiles over $24 \mathrm{~h}$ at Munich airport on 2/3 July 2010.
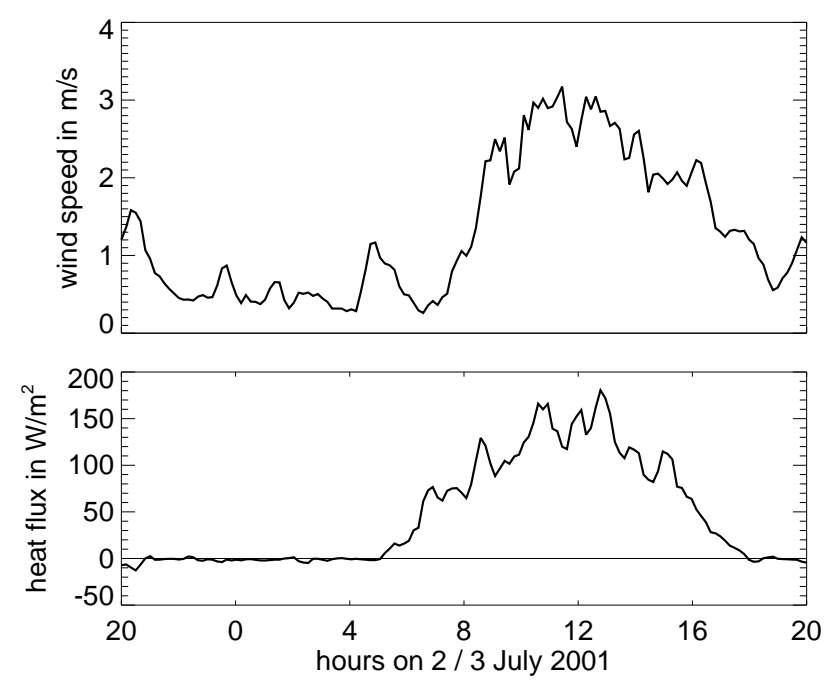

Fig. 9. Wind speed (top) and sensible heat flux (bottom) at $10 \mathrm{~m}$ height at Munich airport on 2/3 July 2010. 

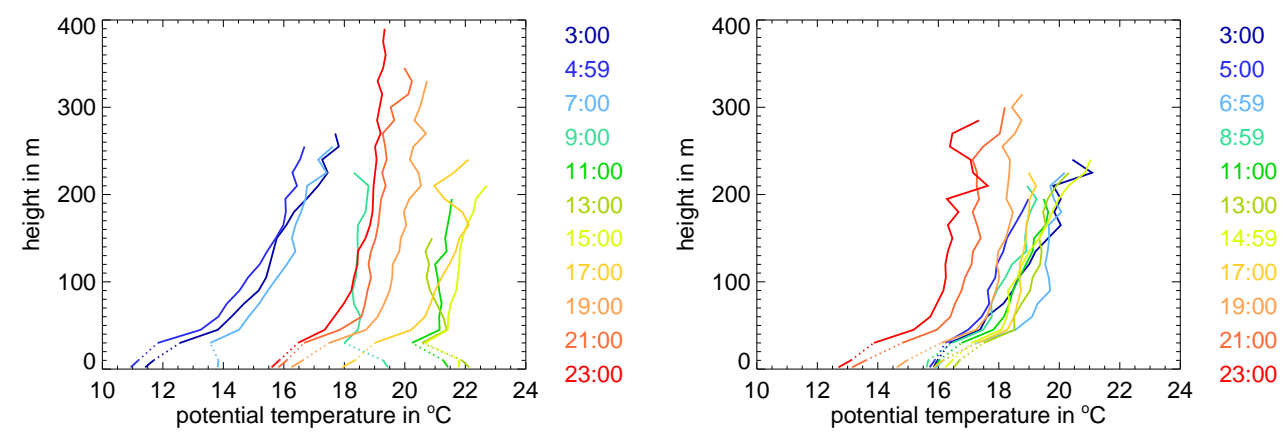

Fig. 10. Development of potential temperature profiles over $24 \mathrm{~h}$ at Jesenice on 21 September 2006 (left) and on 24 October 2006 (right).
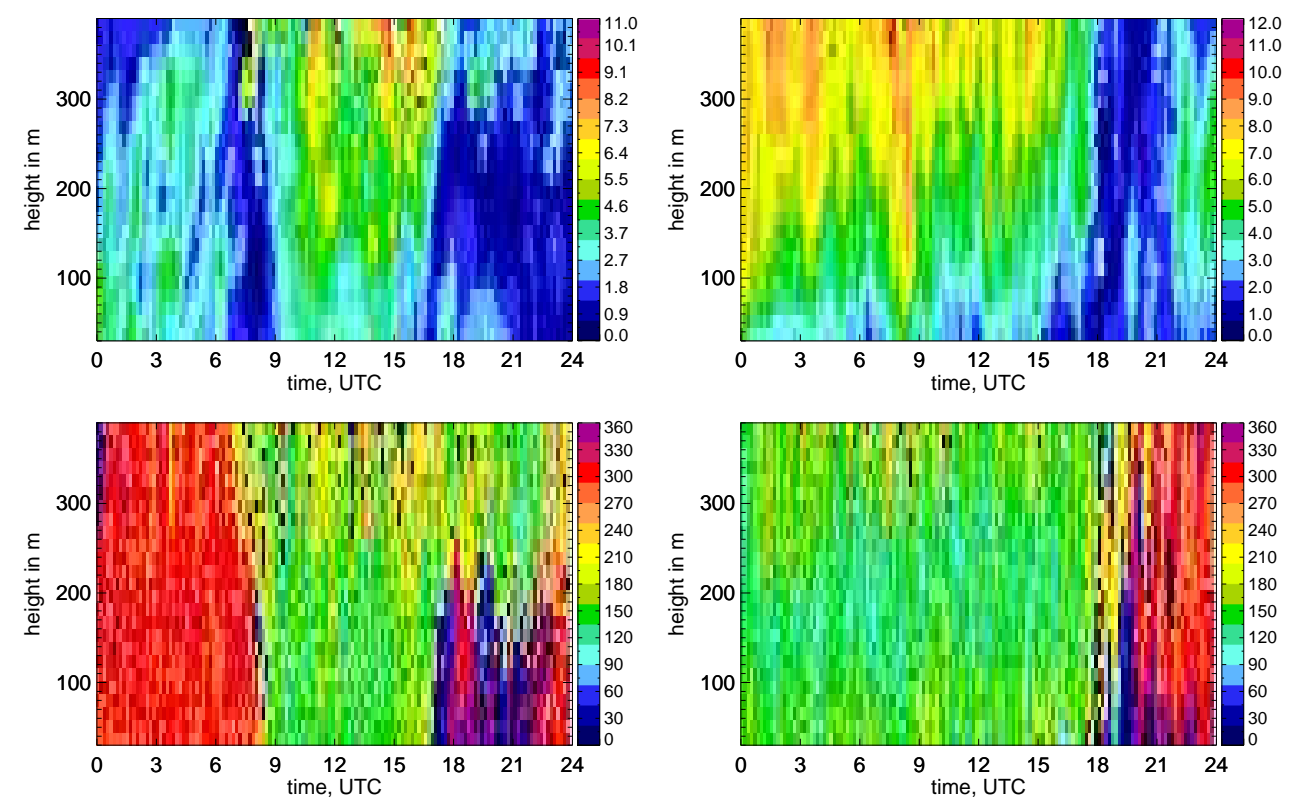

Fig. 11. Wind speed (top) and wind direction (bottom) at Jesenice on 21 September 2006 (left) and on 24 October 2006 (right).

high stability. The depth of the stable layer cannot clearly be determined because it exceeds the range of the RASS. After 05:00 UTC, sensible heat flux increases and warms the layer below $100 \mathrm{~m}$ until 07:00 UTC. With the onset of a stronger wind after 07:00 UTC, a vertical mixing sets on and results in neutral profiles. The measuring range of the RASS is controlled by the combination of wind and turbulence (Kon, $1985)$ and remains lower than $400 \mathrm{~m}$ during daytime. The increased range of $500 \mathrm{~m}$ at 19:00 UTC is probably due to weakening of the wind in combination with residual turbulence.

Unstable temperature profiles often show a mismatch between near-surface temperature and RASS temperature. One reason is probably that the RASS correction retrieval does not include influences of environmental parameters like turbulence and vertical wind. Moreover, the inhomogeneous surface conditions (concrete and grass) may have some effect on the temperature profiles, particularly in case of strong irradiation.

\subsection{Thermal structure of the valley atmosphere}

The second location of RASS measurements is Jesenice in Slovenia. The Jesenice valley runs from $120^{\circ}$ to $300^{\circ}$ and the crest reaches up to $1000 \mathrm{~m}$ over the valley floor. The meteorological situation is dominated either by a thermally induced mountain-valley wind regime in calm and sunny situations or by a dynamically induced flow through the valley in windy and overcast situations. The thermal structures of the valley atmosphere differ strongly between these situations.

Figure 10 (left) shows the temperature profiles during $24 \mathrm{~h}$ in a mountain-valley wind regime. While the morning and evening profiles exhibit stable stratification, the profiles between 09:00 and 15:00 UTC are adiabatic. In contrast to the profile development over flat terrain (see Fig. 8), there is a rapid change in the regimes between 07:00 and 09:00 UTC and between 17:00 and 19:00 UTC. Warming and cooling is not coming from the surface but is controlled by advection 

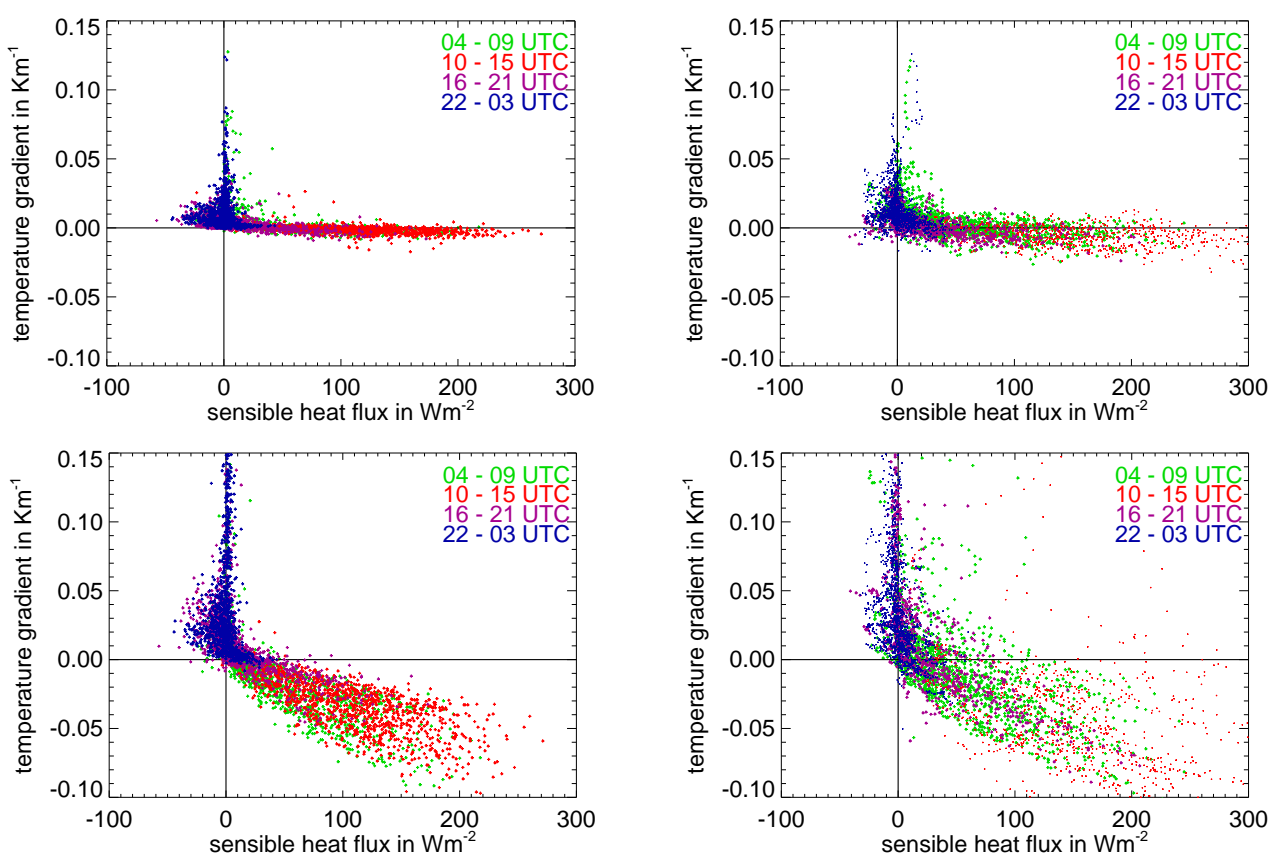

Fig. 12. Temperature gradients near the surface (lower panels) and at elevated height (upper panels) versus sensible heat flux at Billwerder (left panels) and Toulouse (right panels). Temperature gradients near the surface: $2 \mathrm{~m}-10 \mathrm{~m}$, at elevated height: $50 \mathrm{~m}-70 \mathrm{~m}$ (Billwerder) and $40 \mathrm{~m}-60 \mathrm{~m}$ (Toulouse), sensible heat flux: $10 \mathrm{~m}$.

and is happening simultaneously over the whole lower valley atmosphere with the onset of another wind regime.

This is illustrated by the time-height plots of wind speed and wind direction in Fig. 11. During the night time, rather weak wind from 300 to $360^{\circ}$ dominates. At about 06:00 UTC the valley wind sets on in $400 \mathrm{~m}$ and reaches the valley floor at about 08:30 UTC. In this time interval, a strong warming can be recognized in the temperature profiles together with a mixing also due to larger wind speeds. At about 17:00 UTC, the mountain wind sets on together with a stabilization of the temperature profiles.

A prevailing dynamical wind regime from 120 to $150^{\circ}$ ("into the valley") can be observed on 24 October 2006 (Fig. 11, left). The temperature profiles (Fig. 10, right) show stable stratification and do not change much over the day until 19:00 UTC. Between 19:00 and 21:00 UTC the temperature profile shifts by about $2 \mathrm{~K}$ to lower values over a height range of at least $300 \mathrm{~m}$. This coincides with a change of the flow from "into the valley" to "out of the valley".

Thus the different wind regimes in a deep valley are accompanied by specific thermal regimes. As the stability in the lowest $300 \mathrm{~m}$ is crucial for air pollution issues, reliable temperature profile measurements are quite important.

A detailed look at the composed temperature profiles also shows a mismatch between the $10 \mathrm{~m}$ temperature and the lowest RASS temperature during unstable conditions. For this location the correction should have been larger. The reason for this is unclear.

\subsection{Flux-gradient studies}

During unstable conditions a linear correlation between the near-surface gradient of potential temperature and the surface sensible heat flux is expected. At larger heights, where convective mixing dominates, the local flux-gradient relation is no longer valid (Arya, 2005). The temperature profiles are close to neutral. During stable conditions the flux-gradient relations near the surface and aloft are much more complicated and are not considered here in more detail.

As an example for flux-gradient relationships, temperature gradients at different heights and sensible heat fluxes are shown for two flat sites. The instrumented tower of the University of Hamburg (http://wettermast-hamburg.zmaw.de/) is situated at Hamburg-Billwerder east of the city in the estuarine flat of the Elbe river. Temperature and heat flux data at heights from $2 \mathrm{~m}$ to $250 \mathrm{~m}$ are available for May and June 2003 with a time resolution of $10 \mathrm{~min}$. At Toulouse (France) a measuring campaign with RASS (lowest measuring height is $40 \mathrm{~m}$ ) and near-surface sonic $(10 \mathrm{~m})$ and profile measurements $(2 \mathrm{~m}$ and $10 \mathrm{~m})$ took place in an undisturbed environment from 1 May to 30 June 2005. Time resolution is 10 min.

The results can be seen in Fig. 12. At both sites the gradients at elevated heights are plotted against $10 \mathrm{~m}$ heat fluxes, because at Toulouse no other heights were available. The Hamburg-Billwerder data were used for a comparison of gradient-flux relations at various heights using gradients and 
fluxes from equal heights on one hand and fluxes from $10 \mathrm{~m}$ only on the other hand. No significant differences were observed.

A clear flux-gradient relation can be seen for positive heat fluxes at both sites in the lower panels of Fig. 12, representing the near-surface layer. The upper panels of Fig. 12 show the corresponding gradients measured at higher levels $(50 \mathrm{~m}$ to $70 \mathrm{~m}$ in Billwerder, $40 \mathrm{~m}$ to $60 \mathrm{~m}$ in Toulouse). In Billwerder the temperature profiles are derived from in-situ sensors installed at the mast, whereas the upper level measurements in Toulouse were based on RASS. We see that the flux-gradient correlation becomes similarly weak at both sites. The RASS data show more scatter of the temperature gradient in the unstable branch which can readily explained by the influence of turbulence on the retrieved RASS temperature (no vertical wind correction has been applied). But apart from this difference the general structure of the regression is very similar to the in-situ data of Billwerder, which confirms the potential of RASS to provide realistic temperature gradients. Without an empirical bi-static correction the temperature gradient at 40-60 m would indicate stable rather than neutral conditions, as shown in Fig. 4.

\section{Conclusions}

Temperature profiles measured by bi-static Doppler-RASS exhibit a bias in the near range, if the deviation from backscatter geometry is neglected. The correction, according to Kon (1981), considered here takes into account the near field of the antennas, but it includes approximations and simplifications which prevent the immediate application of the correction scheme. We studied the possibility of semiempirical tuning of the effective antenna apertures in order to achieve best agreement with reality. For the considered RASS system an effective aperture radius of 0.8 of the physical radius was found to be optimal. This has been confirmed by comparison with near-surface in-situ measurements and evaluation of temperature profiles under presumably adiabatic conditions. A variation of the tuning parameter $a_{e}$ consistently shows the most plausible results for $a_{e}=0.8$.

Due to the lack of simultaneous RASS and mast measurements, the correction of the temperature profiles is based on plausibility assumptions for near neutral stability conditions. This procedure is chosen because neutral temperature profiles are well known, even though the performance of RASS is not optimal (influence of turbulence and vertical wind) in such conditions. Although the insufficient correction of the lowest height level during unstable conditions, observed in Jesenice, is an unexplained deficiency, the overall quality of RASS temperature profiles in the lowest $300 \mathrm{~m}$ has been improved significantly.

The potential of Doppler-RASS in combination with SODAR for continuously monitoring the diurnal development of the thermal and dynamic boundary layer structure in flat and complex topography as well as for the assessment of flux-gradient relations has been demonstrated by field measurements. The main operation fields of RASS are the investigation and monitoring of inversion height and stratification of the lowest $300 \mathrm{~m}$ for air pollution and diffusion problems. At least for these applications, the presented empirical bistatic correction of temperature profiles is a benefit.

Edited by: P. Stammes

\section{References}

Angevine, W., Bakwin, P., and Davies, K.: Wind profiler and RASS measurements compared with measurements from a 450-m-tall tower, J. Atmos. Ocean.-Tech., 15, 818-825, 1998.

Argentini, S., Pietroni, I., Gariazzo, C., Conidi, A., Mastrantonio, G., Pelliccioni, A., Petenko, I., Viola, A., and Amicarelli, A.: Temperature profiles by ground-based remote sensing and in-situ measurements, IOP Conf. Series: Earth and Environment Science, 1, 1-9, 2008.

Argentini, S., Pietroni, I., Gariazzo, C., Amicarelli, A., Mastrantonio, G., Pelliccioni, A., Petenko, I., and Viola, A.: Boundary layer temperature profiles by a RASS and a microwave radiometer: Differences, limits and advantages, IL NUOVO CIMENTO, 124 B, 549-564, 2009.

Arya, S.: Micrometeorology and atmospheric boundary layer, Pure Appl. Geophys., 162, 1721-1745, 2005.

Bonino, G., Elisei, G., Marzorati, A., and Trivero, P.: Results on planetary boundary layer sounding by automatic RASS, Atmos. Res., 20, 309-316, 1985.

Görsdorf, U.: About the accuracy of temperature measurements with rass, Meteorol. Z., 7, 241-247, 1998.

Görsdorf, U. and Lehmann, V.: Enhanced accuracy of RASSmeasured temperatures due to an improved range correction, J. Atmos. Ocean.-Tech., 17, 406-416, 2000.

Kaimal, J. C. and Gaynor, J. E.: Another look at sonic thermometry, Bound.-Lay. Meteorol., 57, 401-410, 1991.

Kallistratova, M. A. and Kon, A. I.: Radio Acoustic Sounding of Atmosphere, Moscow, Nauka, 196 pp., 1985.

Kon, A. I.: A bistatic radar-acoustic atmospheric sounding system, Izv. Atmos. Ocean. Phys. (Engl. Transl.), 17, 481-484, 1981.

Kon, A. I.: Combined effect of turbulence and wind on the signal intensity in radio-acoustic sounding of the atmosphere, Izv. Atmos. Ocean. Phys. (Engl. Transl.), 21, 942-947, 1985.

Kon, A. I. and Tatarskii, V. I.: The scattered signal frequency spectrum for radioacoustical atmospheric soundings (English translation), Izv. Acad. Sci. USSR, Atmos. Ocean. Phys. (Engl. Transl.), 17, 142-148, 1980.

Lataitis, R.: Theory and application of a radio-acoustic sounding system, Technical report, Ph.D. Thesis, University of Colorado, Boulder, Colorado, USA, 1992.

Lataitis, R., Strauch, R. G., and Moran, K. P.: Temperature errors in rass caused by winds and turbulence, Proc. 26th Intl. Conf. on Radar Meteorol., 24-28 May 1993, Norman, OK, USA, 1993.

Makarova, T.: Measurement of temperature profiles by radioacoustic sounding, Izv. Akad. Nauk, USSR, Physics Atm. Ocean., 16, 118-120, 1980. 
Marshall, J. M., Peterson, A. M., and A. A. Barnes, J.: Combined radar-acoustic sounding system, Appl. Optics, 11, 108$112,1972$.

Nalbandian, O.: For the theory of radio-acoustic sounding of the atmosphere, Izv. Akad. Nauk, USSR, Physics Atm. Ocean., 13, 245-253, 1977.

North, E., Peterson, A., and Parry, H.: RASS, a remote sensing system for measuring low-level temperature profiles, B. Am. Meteorol. Soc., 54, 912-919, 1973.

Pérez, I., García, M., Sánchez, M., and de Torre, B.: Description of atmospheric variables measured with a RASS sodar: Cylces and distribution functions, J. Wind Eng. Ind. Aerod., 96, 436-453, 2008.
Petenko, I. V.: Improved estimation of errors due to antenna geometry in rass based on a radar wind profiler, Meteorol. Atmos. Phys., 71, 69-79, 1999.

Peters, G. and Angevine, W.: On the correction of RASS temperature errors due to turbulence, Contr. Atmos. Physics, 69, 81-96, 1996.

Peters, G., Timmermann, H., and Hinzpeter, H.: Temperature sounding in the planetary boundary layer by RASS-system analysis and results, Int. J. Remote Sens., 4, 49-63, 1983. 